\title{
miR-7 inhibits the invasion and metastasis of gastric cancer cells by suppressing epidermal growth factor receptor expression
}

\author{
JUAN XIE $^{1,2}$, MING CHEN $^{1}$, JING ZHOU ${ }^{1}$, MING-SHU MO ${ }^{2}$, LI-HUI ZHU ${ }^{1}$, \\ YAN-PING LIU ${ }^{1}$, QING-JUN GUI ${ }^{2}$, LI ZHANG ${ }^{1}$ and GUO-QING LI ${ }^{1}$ \\ ${ }^{1}$ Department of Gastroenterology, The Second Affiliated Hospital of the University of South China, Hengyang, Hunan 421001; \\ ${ }^{2}$ Department of Diagnostics, Medical College of the University of South China, Hengyang, Hunan 421001, P.R. China
}

Received December 16, 2013; Accepted February 11, 2014

DOI: $10.3892 /$ or.2014.3052

\begin{abstract}
The present study profiled differentially expressed microRNAs (miRs) in gastric cancer cell lines and then investigated miR-7 expression in gastric cancer tissue specimens and the effects of miR-7 on the growth, invasion and metastasis of gastric cancer cells and the underlying molecular events. A microRNA microarray was used to profile differentially expressed miRNAs in human gastric cancer cell lines relative to a normal stomach mucosal epithelial cell line. The miRNA miR-7 was selected for further investigation, which included real-time reverse-transcription PCR (qRT-PCR) analysis of miR-7 levels in different gastric cancer cell lines and tissues and distant non-tumor tissues from patient resections. Cell Counting Kit-8 (CCK-8), Transwell migration and invasion, and western blot assays were performed to assess tumor cell viability, invasion and gene expression, respectively, after miR-7 transfection. The miRNA microarray profiling revealed 14 upregulated miRNAs (including miR-21, miR-26b and miR-30b) and 19 downregulated miRNAs (including let-7i, miR-7 and miR-622) between gastric cancer and normal cell lines. The qRT-PCR analysis confirmed that reduced miR-7 expression occurred more frequently in poorly and moderately differentiated gastric cancer MGC-803, MKN-45 and SGC-7901 cell lines than in the well-differentiated gastric cancer NCI-N87 cell line, which was consistent with the results for gastric cancer tissues. Expression of miR-7 was downregulated in $86.9 \%(20 / 23)$ of the gastric cancer tissues compared with that in the distant non-tumor tissues. Restoration of miR-7 expression significantly inhibited tumor cell viability, invasiveness and migration when compared with the control cells. Luciferase assay confirmed the epidermal growth factor receptor (EGFR) as a target gene of $\mathrm{mR}-7$, and expression of
\end{abstract}

Correspondence to: Dr Guo-Qing Li, Department of Gastroenterology, The Second Affiliated Hospital of the University of South China, Hengyang, Hunan 421001, P.R. China

E-mail: ligq1970@163.com

Key words: gastric cancer, DNA microarray, miR-7, EGFR, invasion, metastasis
miR-7 significantly suppressed EGFR expression at both the mRNA and protein levels. The data from the present study demonstrated that reduced miR-7 expression contributes to gastric cancer development and progression. Further study will investigate miR-7 in the regulation of EGFR expression in vitro and in vivo.

\section{Introduction}

Human gastric cancer is one of the most common malignancies in the world, with 934,000 new cases occurring each year. The overall 5-year survival rate of gastric cancer remains less than $20 \%$. Advanced disease stages and tumor metastasis contribute to the high mortality rate in patients with gastric cancer (1), which is associated with abnormalities in tumor intracellular signaling networks. During tumor development, environmental and genetic factors interact to contribute to the dysregulation of gene signaling pathways, which include JAK-STAT, Wnt, MAPK and PI3K (2-6).

Studies have shown that abnormal expression of microRNAs (miRNAs, miRs), small non-coding RNAs $\sim 18-22$ nucleotides in length that post-transcriptionally regulate expression of target genes, is one of the key mechanisms in cancer development, invasion and metastasis (7-9). Many studies have shown altered expression of various miRNAs in human carcinogenesis. For example, miR-451, miR-101, let-7 and miR-17-92 have been reported to regulate the invasion and metastasis of tumor cells by targeting the PI3K/Akt and MAPK pathways (10-13).

In gastric cancer, miRNAs have been found to play important roles in tumor initiation and progression, including miR-20, miR-34b, miR-128, miR-129, miR-148, miR-150 and miR-21 (14,15). Other miRNAs (miR-15b, miR-16, miR-372 and miR-150) affected gastric cancer apoptosis and drug resistance (16-18). The miRNAs miR-218, miR-141, miR-375, miR-101, miR-21, miR-449 and miR-107 are implicated in the regulation of gastric cancer cell differentiation, invasion and metastasis (19-25). Further investigation of aberrant miRNA expression in gastric cancer cells may provide insightful information for a better understanding of gastric cancer and the mechanisms underlying gastric carcinogenesis. Such investigations could help us to develop novel strategies for future prevention, diagnosis and treatment of gastric cancer. 
To this end, in the present study we first profiled differentially expressed miRNAs in gastric cancer cell lines relative to a normal stomach mucosal epithelial cell line. We then investigated miR-7 expression in gastric cancer tissue specimens and the effects of miR-7 on growth, invasion and metastasis of gastric cancer cells and the underlying molecular events. The miR-7 gene is localized at the intron region of the heterogeneous ribonucleic protein $\mathrm{k}$ gene on human chromosome 9 , and altered miR-7 expression is associated with the progression of numerous types of tumors since miR-7 can target and inhibit expression of the epidermal growth factor receptor (EGFR), Pak1, Bcl-2 and IGF1R in breast cancer, Schwann cell tumors, tongue squamous cell carcinoma and non-small cell lung cancer (26-29).

\section{Materials and methods}

Cell lines and culture. The human gastric adenocarcinoma cell lines SGC-7901 (moderately differentiated) and MKN-45 (poorly differentiated) and the normal human gastric epithelial cell line GES-1 were obtained from the Cell Bank of Xiangya Central Laboratory, Central South University (Changsha, Hunan, China). The human gastric carcinoma cell lines NCI-N87 (well differentiated) and MGC-803 (poorly differentiated) were obtained from the Cell Bank of the Chinese Academy of Sciences (Shanghai, China).

MGC-803, MKN-45 and SGC-7901 cells were maintained in RPMI-1640 medium. NCI-N87 and GES-1 cells were maintained in Dulbecco's modified Eagle's medium (DMEM; HyClone-Pierce, Logan, UT, USA) supplemented with 10\% fetal bovine serum (FBS; Invitrogen, Carlsbad, CA, USA), penicillin (100 U/ml; Sigma Chemicals, St. Louis, MO, USA), and streptomycin (100 $\mu \mathrm{g} / \mathrm{ml}$; Sigma Chemicals). All cells were cultured in a humidified incubator with $5 \% \mathrm{CO}_{2}$ at $37^{\circ} \mathrm{C}$.

RNA isolation and miRNA microarray profiling. Total cellular RNA was isolated from cells of each cell line using TRIzol reagent (Invitrogen) and purified with an RNeasy Mini kit (Qiagen, Hilden, Germany). After being labeled using the miRCURY Array Power Labeling kit (Exiqon, Vedbaek, Denmark), these RNA samples were used as probes to hybridize with the miRCURY microRNA Array kit (Exiqon) in a Hybridization Chamber II (Ambion, Austin, TX, USA) in accordance with the manufacturer's instructions. Hybridized miRNA microarrays were then scanned using a GenePix 4000B microarray scanner (Axon Instruments, Inc., Union City, CA, USA), and the data were analyzed using GenePix Pro software v6.0 (Axon). Differential miRNA expression (fold change $>1.50$ or ratio $<0.667)$ and cluster analysis were performed using MeV software (v4.6, TIGR). Some of these differentially expressed miRNAs were validated using qRT-PCR.

Gastric cancer tissue specimens. Gastric cancer tissue samples were obtained from 23 patients who underwent partial gastrectomy between March and November 2011 at the First Affiliated Hospital and Second Affiliated Hospital of the University of South China (Hengyang, China). Patients included 12 men aged $60.9 \pm 9$ years and 11 women aged $52.8 \pm 9.5$ years. These patients did not receive any radiotherapy and chemotherapy before surgery.
Gastric cancer tissues and matched non-tumor tissues were obtained from surgical resections and were each divided into two parts: one for pathological diagnosis and another for extracting total RNA. Pathological diagnoses were performed independently by two pathologists in accordance with the WHO standard for pathological diagnosis of gastric cancer. Nine of these cases were histologically diagnosed as well/ moderately differentiated gastric cancers, while 14 were diagnosed as poorly differentiated gastric cancers.

The present study was approved by our institutional review boards of the First and Second Affiliated Hospitals of the University of South China. Each patient provided informed consent before participation in the study.

$q R T-P C R$. Total cellular RNA was isolated from the gastric cell lines and tissues using TRIzol reagent (Invitrogen) and then reverse transcribed to cDNA using a Hairpin-it miRNA qPCR quantitation kit (Shanghai GenePharma Co., Ltd., Shanghai, China) in accordance with the instructions. The PCR primers were: hsa-miR-7 (to generate an 82-bp PCR product), 5'-CCACGTTGGAAGACTAGTGATTT-3' and 5'-TATGGTTGTTCTGCTCTCTGTCTC-3'; human epidermal growth factor receptor (EGFR; to generate a 93-bp PCR product), 5'-AAAGAATACCATGCAGAAGGAGG-3' and 5'-GACATCACTCTGGTGGGTATAGA-3'; U6 (as an internal control, to generate a 70-bp PCR product), 5'-ATTGGAA CGATACAGAGAAGATT-3' and 5'-GGAACGCTTCACGA ATTTG-3', and glyceraldehyde 3-phosphate dehydrogenase (GAPDH, an internal control, to generate a 113-bp PCR product), 5'-CATGAGAAGTATGACAACAGCCT-3' and 5'-AGTCCTTCCACGATACCAAAGT-3'. All of the primers were obtained from Shanghai GenePharma. The qRT-PCR conditions consisted of an initial $95^{\circ} \mathrm{C}$ for $3 \mathrm{~min}$; and 40 cycles of $95^{\circ} \mathrm{C}$ for $30 \mathrm{sec}$ and $62^{\circ} \mathrm{C}$ for $40 \mathrm{sec}$. To generate the melting curves, the PCR amplification was carried out by slowly heating at $0.1^{\circ} \mathrm{C} / \mathrm{sec}$ increments from 60 to $94^{\circ} \mathrm{C}$.

Construction of the miR-7 expression vector and gene transfection. To construct the miR-7 expression vector, we synthesized a mature miR-7 sequence (5'-TGGAAGACTAG TGATTTTGTTGT-3') and inserted these double-stranded oligonucleotides into a linearized PcDNATM6.2-GW/ EmGFP-miR vector (Invitrogen). After amplification, we sequenced this vector to confirm successful cloning. We also constructed a negative control vector using non-sense sequences (5'-AAATGTACTGCGCGTGGAGAC-3') to insert into a pcDNA $6.2-\mathrm{GW} / \mathrm{miR}$ vector. For gene transfection, we grew MGC-803 cells and transfected the above vectors into this cell line using GBfectene-Elite (Genebank Biosciences, Suzhou, China) in accordance with the manufacturer's instructions.

The following experimental groups were created: untreated, transfection reagent only, negative control vector and miR-7 vector. The transfection rate was confirmed using an inverted fluorescence microscope and qRT-PCR $48 \mathrm{~h}$ after transfection.

Cell viability CCK- 8 assay. Cells in each group were suspended and inoculated into 96-wells at 1,000 cells/well. After reaching $80 \%$ confluency, the cells were transfected with plasmids $(0.05 \mu \mathrm{g} /$ well $)$ using GBfectene-Elite in accordance 
with the manufacturer's instructions, and cultured for $72 \mathrm{~h}$. At the end of the experiment, $10 \mu \mathrm{l}$ of the CCK-8 solution (Yiyuan Biotechnology, Guangzhou, China) was added into each well and the cells were further incubated at $37^{\circ} \mathrm{C}$ in a humidified incubator with $5 \% \mathrm{CO}_{2}$ for $1 \mathrm{~h}$. Optical densities were measured at an absorbance of $450 \mathrm{~nm}$ using an ELX800 enzyme-linked immunoassay analyzer (BioTek, Winooski, VT, USA). The average and standard deviation of each group were calculated and the data are presented in a histogram.

Transwell tumor cell migration and invasion assays. A Transwell chamber (PengBo Biotech, Changsha, China) was used to detect gastric cancer cell migration and invasion capacity. For invasion, the membrane in the Transwell chamber was coated with Matrigel basement membrane matrix (BD BioCoat, Bedford, MA, USA). The miR-7-transfected or control vector-transfected cells were suspended at $1.0 \times 10^{6} /$ $\mathrm{ml}$ in DMEM containing 1.0\% BSA. One hundred microliters was added into the upper chamber of the Transwell, and $500 \mu \mathrm{l}$ DMEM containing 20\% FBS was added into the lower chamber. The cells were incubated for $48 \mathrm{~h}$. At the end of the experiments, the cells on the upper side of the membrane were carefully wiped out, and the cells that had migrated or invaded into the lower side of the membrane were fixed with $4 \%$ paraformaldehyde and stained with 4',6-diamidino-2-phenylindole (DAPI). Images were then captured, and the number of cells was counted in 5 random fields for each group and summarized as mean \pm standard deviation (SD) for statistical analysis.

Protein extraction and western blot analysis. Cells were washed 3 times with phosphate-buffered saline (PBS) and lysed in RIPA buffer (Beyotime Institute of Biotechnology, Shanghai, China) to extract the total cellular protein. Equal amounts of protein samples were resolved via $8 \%$ sodium dodecyl sulfate-polyacrylamide gel electrophoresis (SDS-PAGE) and transferred onto a polyvinylidene difluoride (PVDF) membrane (Beyotime Institute of Biotechnology). After blocking with a blocking buffer (GenePharma) for $1 \mathrm{~h}$, the membrane was incubated with a primary antibody against EGFR (Proteintech Group, Chicago, IL, USA) or GADPH (Sigma-Aldrich) at a dilution of 1:500 at $4^{\circ} \mathrm{C}$ overnight. The next day the membranes were washed with PBS thrice and then further incubated with a horseradish peroxidaseconjugated secondary antibody (Jackson ImmunoResearch, West Grove, PA, USA) at a dilution of $1: 1,000$ at $37^{\circ} \mathrm{C}$ for $1 \mathrm{~h}$. After washing with PBS thrice, positive protein bands were visualized using SuperSignal West Pico chemiluminescence substrates (Thermo Fisher Scientific, Rockford, IL, USA). Image analysis was performed with Alpha Imager 2200 (UVP, Upland, CA, USA).

Dual-luciferase reporter gene assay. To assess the direct binding of miR-7 to EFGR cDNA, we performed a luciferase assay. In brief, the cells were co-transfected with EGFR 3'UTR-pmirGLO plasmid and hsa-mir-7 mimic vector or the negative control plasmid hsa-miR-NC using Lipofectamine 2000 (Invitrogen) in accordance with the manufacturer's instructions. Twenty-four or $48 \mathrm{~h}$ after transfection, the cells were prepared for the luciferase assay using the Dualluciferase reporter assay system (Promega, Madison, WI,
USA) in accordance with the kit instructions. Luciferase activity was detected with a GloMax ${ }^{\mathrm{TM}}$ 20/20 Luminometer (Promega). The relative luciferase activity was defined as the ratio of firefly luciferase activity to Renilla luciferase activity. $\Delta$ luciferase activity was used for statistical analysis of data, where $\Delta$ luciferase activity $=($ Firefly $/$ Renilla $)$ sample/(Firefly $/$ Renilla) control.

Statistical analysis. Statistical analyses were performed using the statistical software SPSS 16.0 (SPSS, Inc., Chicago, IL, USA). All data are expressed as mean \pm standard deviation. Differences between groups were analyzed with one-way ANOVA and the least significant difference test; $\mathrm{P}<0.05$ was considered to indicate a statistically significant result.

\section{Results}

Differentially expressed miRNAs in gastric cancer cells. We performed a microRNA microarray assay to identify differentially expressed miRNAs in gastric cancer cell lines (MGC-803, MKN-45, SGC-7901 and NCI-N87) that varied in the degrees of tumor differentiation, as well as in the normal gastric mucosal epithelial cell line GES-1 (Fig. 1A). In these gastric cancer cell lines, we found 14 miRNAs that were overexpressed relative to the normal gastric mucosal GES-1 cells (ratio $>1.5$ between tumor and normal cells). These overexpressed miRNAs were: hsa-miR-23a, hsa-miR-27b, hsa-miR-1908, hsa-miR-26b, hsa-miR-3607-3p, hsa-miR-20a*, hsa-miR-21, hsa-miR-122*, hsa-miR-193a-3p, hsa-miR-26a, hsa-miR-29c, hsa-miR-16, hsa-miR-30b and hsa-miR-3647-3p. In contrast, 19 miRNAs were reduced by 1.5 -fold or more (i.e., ratio $<0.667$ between tumor and normal cells). The miRNAs that were underexpressed were: hsa-miR-1247, hsa-miR-20b*, hsa-miR-4301, hsa-miR-1299, hsa-miR-484, hsa-miR-885-5p, hsa-miR-1265, hsa-miR-589, hsa-miR-550a, let-7i*, hsa-miR-2355-3p, hsa-miR-4284, let-7i, hsa-miR-7, hsa-miR-1260, hsa-miR-622, hsa-miR-185, hsa-miR-378 and hsa-miR-378c.

The miRNA hsa-miR-7 was downregulated in all four gastric cancer cell lines comprising well-, moderately and poorly differentiated gastric cancer cells, relative to normal GES-1 cells. Thus, we chose hsa-miR-7 for further investigation. The underexpression of hsa-miR-7 in the NCI-N87, SGC-7901, MKN-45 and MGC-803 cell lines was confirmed via qRT-PCR (Fig. 1B).

We then assessed miR-7 expression in gastric cancer tissue specimens, and found that relative to the normal tissues, expression of miR-7 was decreased in $86.9 \%$ of gastric cancer tissues. Levels of miR-7 expression were significantly lower in moderately and poorly differentiated cancer tissues when compared with that in the well-differentiated gastric cancer tissues (Fig. 1C and D).

Expression of miR-7 inhibits gastric cancer cell viability and invasion. To further assess the role of miR-7 in gastric cancer, we constructed a miR-7 expression vector for gene transfection and found that the transfection efficiency of the miR-7 plasmid and negative control groups was $>80 \%$ in MGC803 cells after a 48-h transfection, via observation under a fluorescence microscope. The qRT-PCR data showed 

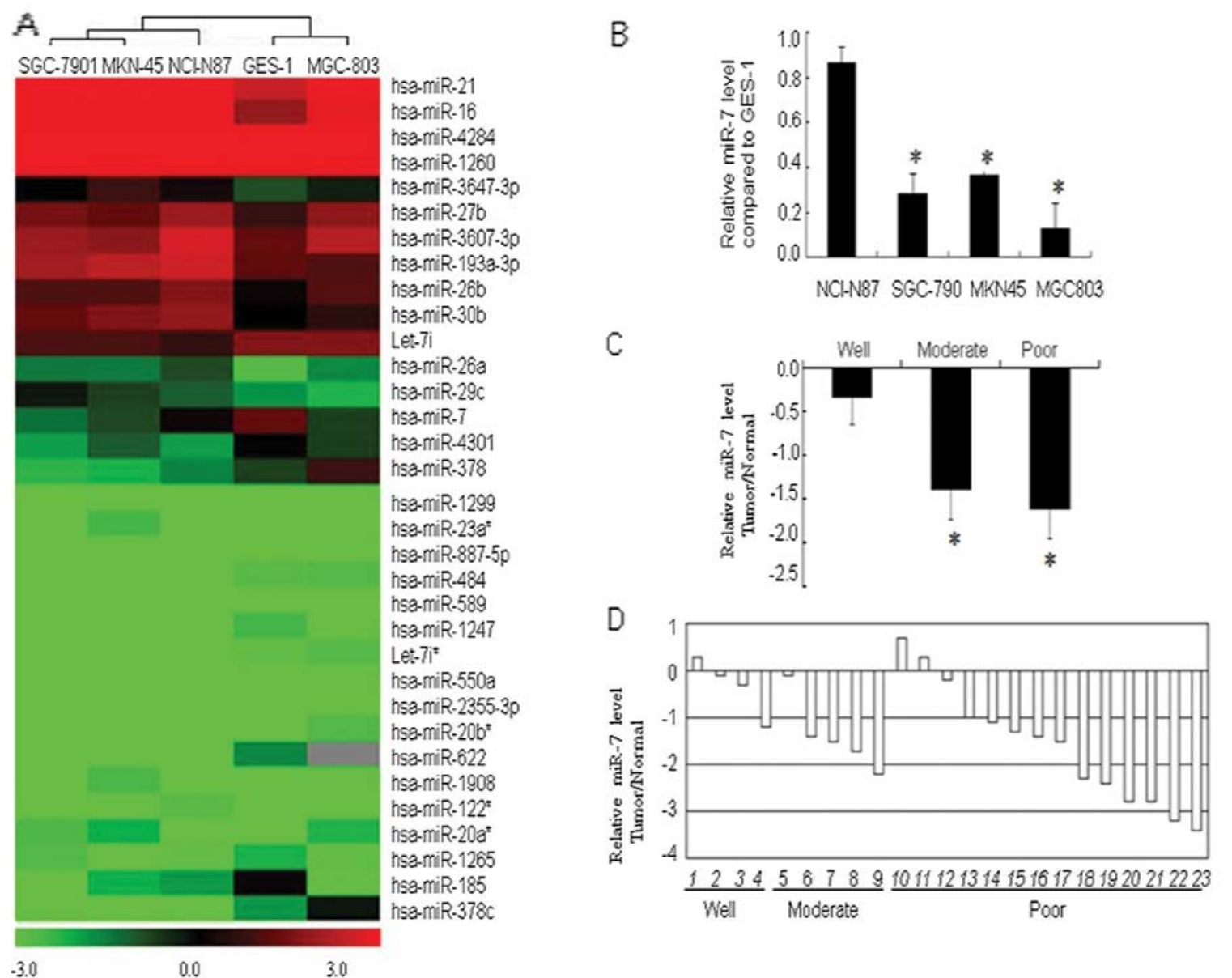

Figure 1. Differential expression of miRNAs in gastric cancer cells and tissues. (A) Cluster analysis of differential miRNA expression in gastric cancer cell lines NCI-N87, SGC-7901, MKN-45 and MGC-803 relative to the normal gastric mucosal GES-1 cells. The color scale shown at the bottom illustrates the relative expression level of an miRNA in a certain slide: red color represents a high relative expression level; green color represents a low relative expression level. (B) Validation of miR-7 expression using qRT-PCR in NCI-N87, SGC-7901, MKN-45, MGC-803 and GES-1 cells, "P<0.05 compared with GES-1 cells (mean $\pm \mathrm{SD} ; \mathrm{n}=3$ ). (C) qRT-PCR analysis of miR-7 in 23 gastric carcinoma and matched adjacent non-tumor tissues. (D) Gastric cancer tissues were grouped according to the level of differentiation: well differentiated $(n=4)$, moderately differentiated $(n=5)$ and poorly differentiated $(n=14)$. A histogram of the miR-7 expression levels in these three tissue groups as indicated at the bottom. ${ }^{*} \mathrm{P}<0.05$ compared with well-differentiated gastric cancer tissues (mean $\pm \mathrm{SD}$; $\mathrm{n}=3$ ).

that levels of miR-7 expression were significantly greater $48 \mathrm{~h}$ after miR-7 plasmid transfection compared with the negative control cells (Fig. 2A). Restoration of miR-7 expression reduced gastric cancer cell viability (Fig. 2B) compared with the controls.

We used a Transwell chamber to detect the effects of miR-7 on the invasion and migration abilities of MGC803 gastric cancer cells. The miR-7-transfected cells exhibited significantly reduced invasion and migration abilities $(33 \pm 11$ and $59 \pm 34$, respectively) compared with the negative control cells $(135 \pm 37$ and $160 \pm 11)$, transfection reagent only cells $(191 \pm 12$ and $183 \pm 23)$, and the parental cells $(149 \pm 39$ and 182 \pm 20 ; Fig. 2C and D).

Expression of the target gene EGFR is inhibited by miR-7. To explore miR-7 targeting (binding) genes, we performed a bioinformatic analysis using TargetScan, PicTar and miRanda software and found that EGFR could be a target gene (Fig. 3A). To confirm whether miR-7 regulates EGFR expression by binding to the EFGR 3'UTR, we performed a dual luciferase reporter assay. The results showed that 24 and $48 \mathrm{~h}$ after co-transfection of EGFR 3'UTR-pmirGLO plasmid and hsa-miR-7, fluorescent activity was significantly reduced when compared to that of the miR-NC control group (Fig. 3D).

We then performed qRT-PCR and western blot analysis and found that levels of EGFR mRNA and protein were significantly less in the miR-7-transfected cells when compared with the levels in the negative control, transfection reagent only cells and the parental cells (Fig. 3B and C). These data suggest that miR-7-induced inhibition of gastric cancer viability and invasion acts through degradation of EGFR mRNA and a decrease in EGFR protein expression.

\section{Discussion}

In the present study, we first profiled differentially expressed miRNAs in gastric cancer cells relative to normal cells, since a great number of studies have shown that altered miRNA expression is closely associated with gastric cancer development and progression (14-25). Indeed, our data revealed a group of miRNAs that were either upregulated or downregulated in cells of the gastric cancer cell lines of various differentiation levels relative to cells of the normal mucosal 


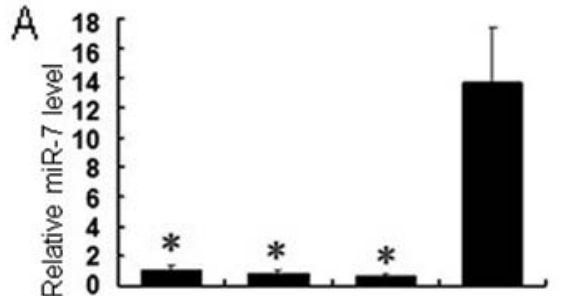

Untreated Reagent Negative miR-7

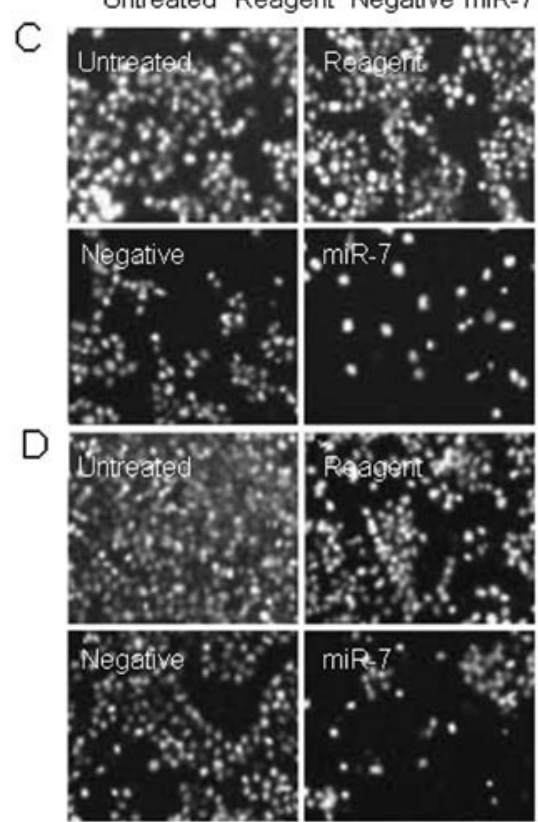

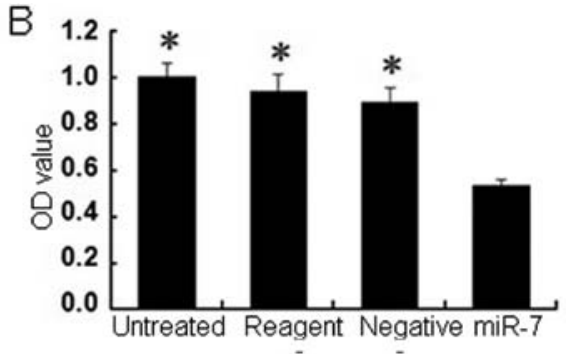
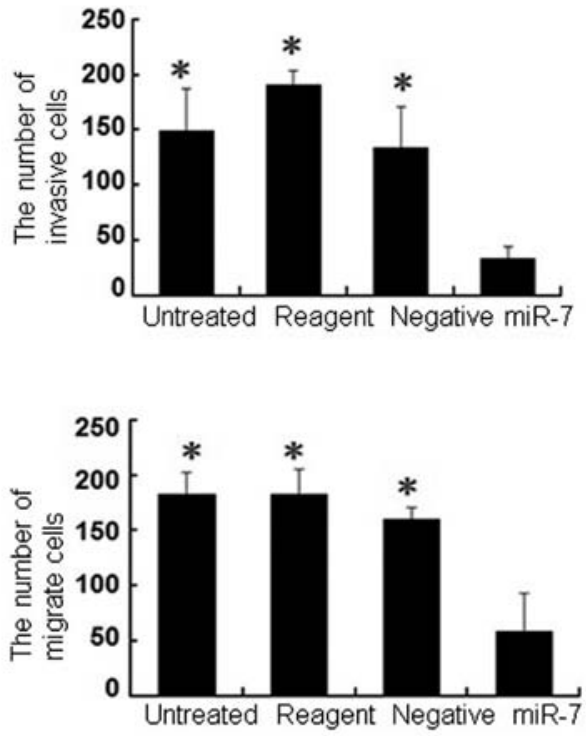

Figure 2. miR-7 inhibits MGC-803 cell viability, and migratory and invasive abilities. (A) qRT-PCR analysis of miR-7 expression. Cells were grown and transfected with miR-7 or the negative control vector or treated with the transfection reagent for $48 \mathrm{~h}$. The transfected cell groups, transfection reagent only cells and the parental cells were then subjected to qRT-PCR. (B) Cell viability CCK-8 assay. Cells were grown and transfected with miR-7 or negative control vector, or transfection reagent, for $72 \mathrm{~h}$. The transfected cell groups, transfection reagent only cells and the parental cells were then subjected to CCK-8 assay. ${ }^{*} \mathrm{P}<0.05$ compared with miR-7-transfected cells (mean $\pm \mathrm{SD} ; \mathrm{n}=3$ ). (C) Transwell tumor cell invasion assay. The respective cells were subjected to the Transwell invasion assay. (D) Transwell tumor cell migration assay. After $48 \mathrm{~h}$ of plasmid transfection, the cells were subjected to Transwell tumor cell migration assay.

cell line. We then focused on miR-7, which was downregulated in all four of the gastric cancer cell lines tested. Consistent with this finding, miR-7 expression was reduced in the gastric cancer tissues, particularly in moderately and poorly differentiated gastric cancer tissues compared to well-differentiated tumors. Furthermore, restoration of miR-7 expression inhibited tumor cell viability, migration and invasion ability. At the gene level, miR-7 was able to bind to the EGFR 3'UTR and suppress expression of EGFR mRNA and EGFR protein. The data from the present study indicate that miRNA expression was altered in gastric cancer cells and tissue specimens and that restoration of miR-7 expression inhibited gastric cancer cell viability, migration and invasion. These results suggest the role of aberrant miR-7 expression in gastric cancer progression and the underlying molecular pathways.

Previous studies have shown that differential expression of various miRNAs not only contributes to carcinogenesis and cancer progression, but can also predict the histological type of cancer, drug resistance and clinical outcomes (7). One study utilized 200 miRNAs to molecularly classify human cancers (30), which may help clinicians to more effectively treat cancer patients. However, to date, there has been no miRNA expression profile study in gastric cancer, while an individual miRNA study showed controversial data (31). In the present study, we selected gastric cancer cell lines with different degrees of differentiation (i.e., well, moderate and poor) relative to normal gastric mucosal cells, to profile aberrant expression of miRNAs and then verify them in gastric cancer cell lines and tissue samples. The purpose of this approach was to identify miRNAs that may be related to gastric cancer progression (such as high metastatic potential). Our data showed that compared with normal gastric mucosal cells, 14 miRNAs were upregulated, whereas 19 miRNAs were downregulated in the four gastric cancer cell lines tested. The altered expression of miR-7, miR-16, miR-21, hsa-miR-30b, let-7i, hsa-miR-622 and hsa-miR-378 found in the present study was consistent with the findings of others $(14,32-37)$, yet not the altered expression of miR-185 and miR-29c $(22,38)$. However, there have been no previous reports of the differential expression of miR-27b, miR-378c and miR-484 in gastric cancer cells. Although data regarding aberrant expression of miR-124, miR-126 and miR-204 have been reported for gastric cancer cells (39-41), the present study did not find any alterations in gastric cancer cell lines. This may be because of differences in the cell lines tested, particularly in the level of cancer cell differentiation and the screening criteria for differential miRNA expression. 
A

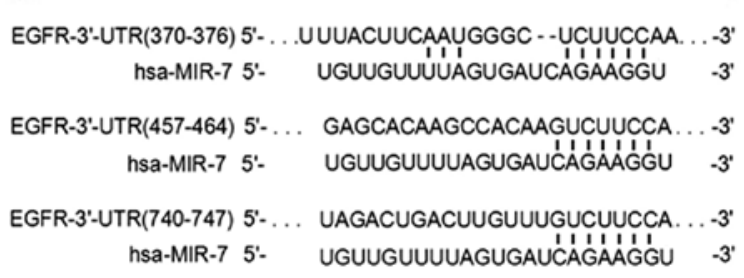

$\mathrm{C}$

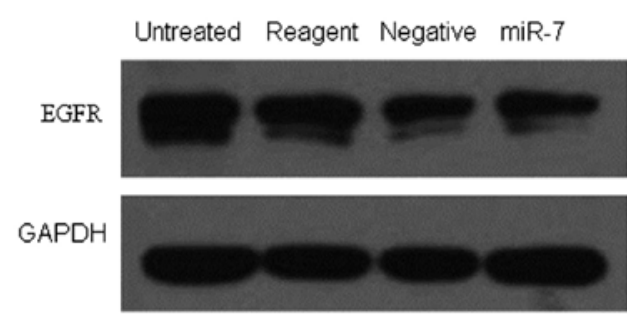

D

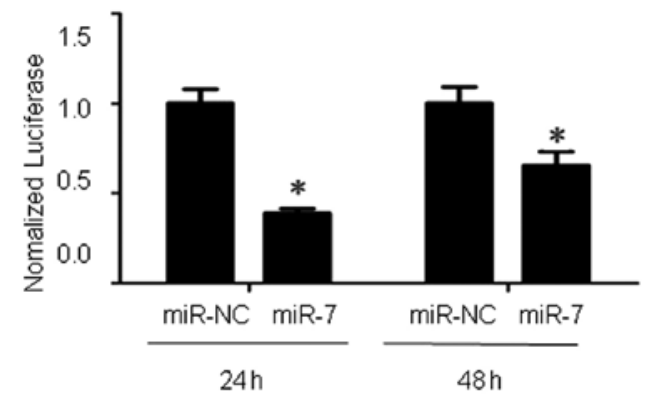

B
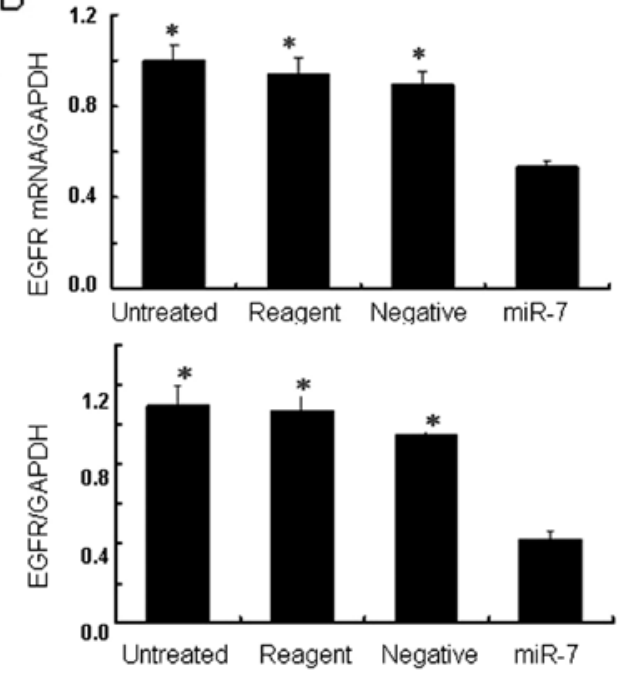

Figure 3. miR-7 inhibits EGFR expression in MGC-803 cells. (A) Potential targeting site of miR-7 on EGFR cDNA as predicted by TargetScan, PicTar and miRanda software. (B) qRT-PCR analysis of EGFR expression. Cells were grown and transfected with miR-7 or negative control vector, or transfection reagent, for $48 \mathrm{~h}$. The transfected cell groups, transfection reagent only cells and the parental cells were then subjected to qRT-PCR. (C) Western blot analysis; $48 \mathrm{~h}$ after transfection of plasmids, cells were subjected to protein extraction and western blot analysis. * $\mathrm{P}<0.05 \mathrm{compared}$ with miR-7 (mean $\pm \mathrm{SD}$; $\mathrm{n}=3$ ). (D) Luciferase assay. Cells were co-transfected with EGFR 3'UTR-pmirGLO and either miR-7 or miR-NC plasmid for 24 and 48 h and subjected to luciferase assay. ${ }^{*} \mathrm{P}<0.05$ compared with miR-NC-transfected cells (mean $\pm \mathrm{SD} ; \mathrm{n}=3$ ).

The miRNA microarray and RT-PCR data of the present study showed that miR-7 expression was significantly lower in the NCI-N87, SGC-7901, MGC-803 and MKN-45 cell lines, which was further confirmed in gastric carcinoma tissues and matched adjacent non-tumor tissues. Restoration of miR-7 expression reduced gastric cancer cell viability, migration and invasion ability. Indeed, the current emphasis regarding the clinical control of human cancer concerns the suppression of cancer cell proliferation, invasion and metastasis, all of which affect tumor classification, treatment and clinical outcomes. Poorly differentiated gastric cancer is more aggressive and is defined by increased proliferation, invasion and metastasis (42), making this type of cancer difficult to treat resulting in a poor patient prognosis. It has been reported that transfection of miR-7 precursor into human gastric cancer AZ521 and Kato III cell lines significantly inhibited gastric cancer cell proliferation (33). Overexpression of miR-7 in the gastric cancer GC9811-P cell line suppressed tumor cell migration and invasion (43). This study is consistent with these previous studies, although different tumor cell lines were used. These data together indicate that miR-7 may play an important role in gastric cancer progression.

Molecularly, miR-7 has been shown to downregulate EGFR expression in glioma, and cancers of the prostate, lung, and breast $(44,45)$, but there has been no previous report of the role of miR-7 in the regulation of EGFR expression in gastric cancer.
As we know, the expression of miRNAs are tissue-specific and time dependent, with different tissues or cells at different stages of biological development expressing various miRNAs (8). In the present study, we performed a bioinformatic analysis and predicted that EGFR cDNA contains hsa-MIR-7 targeting sequences. We then performed a luciferase assay to confirm the interaction between miR-7 and the EGFR 3'UTR in human gastric cancer cell lines. Western blot analysis and qRT-PCR data further revealed that miR-7 suppressed levels of EGFR mRNA and protein in human gastric cancer cell lines.

EGFR is involved in the regulation of tumor cell proliferation, angiogenesis, invasion, metastasis and inhibition of apoptosis through downstream signal transduction pathways, and is closely correlated with tumor initiation, progression and poor prognosis of gastric cancer (46-52). The downstream of EGFR signal transduction pathways include the Ras/Raf/ MEK/ERK-MAPK and PI3K/Akt/mTOR pathways $(4,53)$. The ERK-MAPK signaling pathway was found to induce cell tumor proliferation by inactivating cell cycle inhibitory protein p27KIP via phosphorylation (54) or by activating matrix metalloproteinases to enhance hydrolysis of the extracellular matrix and invasion of tumor cells (55), in addition to participation in the regulation of apoptosis of tumor cells. Furthermore, the PI3K/Akt pathway can activate mTOR signaling networks to promote translation of key proteins for cell growth $(56,57)$. 
The present study is proof-of-principle and future studies will be carried out to investigate these gene pathways in the control of gastric cancer development and progression. We will explore whether miR-7 offers a novel strategy for gastric cancer therapy.

\section{Acknowledgements}

We thank Dr Xiaoyong Lei of the Life Science and Technology College of the University of South China, Dr Rongfang $\mathrm{He}$ of the Pathology Department of the First Affiliated Hospital of the University of South China and Dr Yong Fang of the Pathology Department of the Second Affiliated Hospital of the University of South China for their technical support and for providing tissue specimens for the present study. We also thank Medjaden Bioscience Ltd., Hong Kong, China, for assisting in the editing of this manuscript. The study was supported in part by a grant from the National Natural Science Foundation of China (\#81071965).

\section{References}

1. Jemal A, Bray F, Center MM, Ferlay J, Ward E and Forman D Global cancer statistics. CA Cancer J Clin 61: 69-90, 2011.

2. Ben-Chetrit N, Tarcic G and Yarden Y: ERK-ERF-EGR1, a novel switch underlying acquisition of a motile phenotype. Cell Adh Migr 7: 33-37, 2013.

3. Thu KL, Radulovich N, Becker-Santos DD, et al: SOX15 is a candidate tumor suppressor in pancreatic cancer with a potential role in Wnt/B-catenin signaling. Oncogene 33: 279-288, 2014.

4. Hill KS, Erdogan E, Khoor A, et al: Protein kinase C $\alpha$ suppresses Kras-mediated lung tumor formation through activation of a p38 MAPK-TGF $\beta$ signaling axis. Oncogene: Apr 22, 2013 (Epub ahead of print).

5. Nojima M, Suzuki H, Toyota M, et al: Frequent epigenetic inactivation of SFRP genes and constitutive activation of Wnt signaling in gastric cancer. Oncogene 26: 4699-4713, 2007.

6. Xu G, Zhang W, Bertram P, Zheng XF and McLeod H: Pharmacogenomic profiling of the PI3K/PTEN-AKT-mTOR pathway in common human tumors. Int J Oncol 24: 893-900, 2004.

7. Krützfeldt J, Poy MN and Stoffel M: Strategies to determine the biological function of microRNAs. Nat Genet 38: S14-S19, 2006

8. Bartel DP: MicroRNAs: genomics, biogenesis, mechanism, and function. Cell 116: 281-297, 2004.

9. Carthew RW: Gene regulation by microRNAs. Curr Opin Genet Dev 16: 203-208, 2006.

10. Hayashita Y, Osada H, Tatematsu Y, et al: A polycistronic microRNA cluster, miR-17-92, is overexpressed in human lung cancers and enhances cell proliferation. Cancer Res 65 : 9628-9632, 2005.

11. Lee YS and Dutta A: The tumor suppressor microRNA let-7 represses the HMGA2 oncogene. Genes Dev 21: 1025-1030, 2007.

12. Nan Y, Han L, Zhang A, et al: miRNA-451 plays a role as tumor suppressor in human glioma cells. Brain Res 1359: 14-21, 2010.

13. Zhang JG, Guo JF, Liu DL, Liu Q and Wang JJ: MicroRNA-101 exerts tumor-suppressive functions in non-small cell lung cancer through directly targeting enhancer of zeste homolog 2 . J Thorac Oncol 6: 671-678, 2011 .

14. Chan SH, Wu CW, Li AF, Chi CW and Lin WC: miR-21 microRNA expression in human gastric carcinomas and its clinical association. Anticancer Res 28: 907-911, 2008.

15. Katada T, Ishiguro H, Kuwabara Y, et al: microRNA expression profile in undifferentiated gastric cancer. Int J Oncol 34: 537-542, 2009.

16. Xia L, Zhang D, Du R, et al: miR-15b and miR-16 modulate multidrug resistance by targeting BCL2 in human gastric cancer cells. Int J Cancer 123: 372-379, 2008.

17. Cho WJ, Shin JM, Kim JS, et al: miR-372 regulates cell cycle and apoptosis of ags human gastric cancer cell line through direct regulation of LATS2. Mol Cells 28: 521-527, 2009.

18. Wu Q, Jin H, Yang Z, et al: MiR-150 promotes gastric cancer proliferation by negatively regulating the pro-apoptotic gene EGR2. Biochem Biophys Res Commun 392: 340-345, 2010.
19. Varambally S, Cao Q, Mani RS, et al: Genomic loss of microRNA101 leads to overexpression of histone methyltransferase EZH2 in cancer. Science 322: 1695-1699, 2008.

20. Zhang Z, Li Z, Gao C, et al: $\mathrm{miR}-21$ plays a pivotal role in gastric cancer pathogenesis and progression. Lab Invest 88: 1358-1366, 2008.

21. Du Y, Xu Y, Ding L, Yao H, Yu H, Zhou T and Si J: Downregulation of miR-141 in gastric cancer and its involvement in cell growth. J Gastroenterol 44: 556-561, 2009.

22. Takahashi Y, Forrest AR, Maeno E, Hashimoto T, Daub CO and Yasuda J: miR-107 and miR-185 can induce cell cycle arrest in human non small cell lung cancer cell lines. PLoS One 4: e6677, 2009.

23. Ding L, Xu Y, Zhang W, et al: MiR-375 frequently downregulated in gastric cancer inhibits cell proliferation by targeting JAK2. Cell Res 20: 784-793, 2010

24. Tie J, Pan Y, Zhao L, et al: MiR-218 inhibits invasion and metastasis of gastric cancer by targeting the Robol receptor. PLoS Genet 6: e1000879, 2010.

25. BouKheir T, Futoma-Kazmierczak E, Jacobsen A, et al: miR-449 inhibits cell proliferation and is down-regulated in gastric cancer. Mol Cancer 10: 29, 2011.

26. Reddy SD, Ohshiro K, Rayala SK and Kumar R: MicroRNA-7, a homeobox D10 target, inhibits p21-activated kinase 1 and regulates its functions. Cancer Res 68: 8195-8200, 2008.

27. Saydam O, Senol O, Würdinger T, et al: miRNA-7 attenuation in Schwannoma tumors stimulates growth by upregulating three oncogenic signaling pathways. Cancer Res 71: 852-861, 2011.

28. Xiong S, Zheng Y, Jiang P, Liu R, Liu X and Chu Y: MicroRNA-7 inhibits the growth of human non-small cell lung cancer A549 cells through targeting BCL-2. Int J Biol Sci 7: 805-814, 2011.

29. Jiang L, Liu X, Chen Z, et al: MicroRNA-7 targets IGF1R (insulin-like growth factor 1 receptor) in tongue squamous cell carcinoma cells. Biochem J 432: 199-205, 2010.

30. Lu J, Getz G, Miska EA, et al: MicroRNA expression profiles classify human cancers. Nature 435: 834-838, 2005.

31. Yao Y, Suo AL, Li ZF, et al: MicroRNA profiling of human gastric cancer. Mol Med Rep 2: 963-970, 2009.

32. Guo XB, Jing CQ, Li LP, et al: Down-regulation of miR-622 in gastric cancer promotes cellular invasion and tumor metastasis by targeting ING1 gene. World J Gastroenterol 17: 1895-1902, 2011.

33. Kong D, Piao YS, Yamashita S, et al: Inflammation-induced repression of tumor suppressor miR-7 in gastric tumor cells. Oncogene 31: 3949-3960, 2012.

34. Shin VY, Jin H, Ng EK, et al: NF-кB targets miR-16 and miR-21 in gastric cancer: involvement of prostaglandin $\mathrm{E}$ receptors. Carcinogenesis 32: 240-245, 2011.

35. Inoue $\mathrm{T}$, Iinuma $\mathrm{H}$, Ogawa $\mathrm{E}$, Inaba $\mathrm{T}$ and Fukushima $\mathrm{R}$ : Clinicopathological and prognostic significance of microRNA107 and its relationship to DICER1 mRNA expression in gastric cancer. Oncol Rep 27: 1759-1764, 2012.

36. Liu K, Qian T, Tang L, Wang J, Yang H and Ren J: Decreased expression of microRNA let-7i and its association with chemotherapeutic response in human gastric cancer. World J Surg Oncol 10: 225, 2012

37. Deng H, Guo Y, Song H, et al: MicroRNA-195 and microRNA378 mediate tumor growth suppression by epigenetical regulation in gastric cancer. Gene 518: 351-359, 2013.

38. Matsuo M, Nakada C, Tsukamoto Y, et al: MiR-29c is downregulated in gastric carcinomas and regulates cell proliferation by targeting RCC2. Mol Cancer 12: 15, 2013.

39. Xia $\mathrm{J}, \mathrm{Wu} \mathrm{Z}, \mathrm{Yu} \mathrm{C}$, et al: $\mathrm{miR}-124$ inhibits cell proliferation in gastric cancer through down-regulation of SPHK1. J Pathol 227: 470-480, 2012

40. Feng R, Chen X, Yu Y, et al: miR-126 functions as a tumour suppressor in human gastric cancer. Cancer Lett 298: 50-63, 2010.

41. Sacconi A, Biagioni F, Canu V, et al: miR-204 targets Bcl-2 expression and enhances responsiveness of gastric cancer. Cell Death Dis 3: e423, 2012.

42. Catalano V, Labianca R, Beretta GD, Gatta G, de Braud F and Van Cutsem E: Gastric cancer. Crit Rev Oncol Hematol 71: 127-164, 2009.

43. Zhao X, Dou W, He L, et al: MicroRNA-7 functions as an antimetastatic microRNA in gastric cancer by targeting insulin-like growth factor-1 receptor. Oncogene 32: 1363-1372, 2013.

44. Webster RJ, Giles KM, Price KJ, Zhang PM, Mattick JS and Leedman PJ: Regulation of epidermal growth factor receptor signaling in human cancer cells by microRNA-7. J Biol Chem 284: 5731-5741, 2009. 
45. Giles KM, Barker A, Zhang PM, Epis MR and Leedman PJ: MicroRNA regulation of growth factor receptor signaling in human cancer cells. Methods Mol Biol 676: 147-163, 2011.

46. Nicholson RI, Gee JM and Harper ME: EGFR and cancer prognosis. Eur J Cancer 37: S9-S15, 2001

47. Saif MW: Colorectal cancer in review: the role of the EGFR pathway. Expert Opin Investig Drugs 19: 357-369, 2010.

48. Lieto E, Ferraraccio F, Orditura M, et al: Expression of vascular endothelial growth factor (VEGF) and epidermal growth factor receptor $(E G F R)$ is an independent prognostic indicator of worse outcome in gastric cancer patients. Ann Surg Oncol 15: 69-79, 2008.

49. Arkenau HT: Gastric cancer in the era of molecularly targeted agents: current drug development strategies. J Cancer Res Clin Oncol 135: 855-866, 2009.

50. She QB, Solit DB, Ye Q, O'Reilly KE, Lobo J and Rosen N: The BAD protein integrates survival signaling by EGFR/MAPK and PI3K/Akt kinase pathways in PTEN-deficient tumor cells. Cancer Cell 8: 287-297, 2005.

51. Corcoran RB, Ebi H, Turke AB, et al: EGFR-mediated re-activation of MAPK signaling contributes to insensitivity of BRAF mutant colorectal cancers to RAF inhibition with vemurafenib. Cancer Discov 2: 227-235, 2012.
52. Kim MA, Lee HS, Lee HE, Jeon YK, Yang HK and Kim WH: EGFR in gastric carcinomas: prognostic significance of protein overexpression and high gene copy number. Histopathology 52: 738-746, 2008.

53. Toulany $M$, Dittmann $K$, Krüger $M$, Baumann $M$ and Rodemann HP: Radioresistance of K-Ras mutated human tumor cells is mediated through EGFR-dependent activation of PI3K-AKT pathway. Radiother Oncol 76: 143-150, 2005.

54. Donovan JC, Milic A and Slingerland JM: Constitutive MEK/ MAPK activation leads to $\mathrm{p} 27^{\mathrm{Kip} 1}$ deregulation and antiestrogen resistance in human breast cancer cells. J Biol Chem 276: 40888-40895, 2001

55. Stamenkovic I: Matrix metalloproteinases in tumor invasion and metastasis. Semin Cancer Biol 10: 415-433, 2000.

56. Pires MM, Hopkins BD, Saal LH and Parsons RE: Alterations of EGFR, p53 and PTEN that mimic changes found in basallike breast cancer promote transformation of human mammary epithelial cells. Cancer Biol Ther 14: 246-253, 2013.

57. Britschgi A, Bill A, Brinkhaus $\mathrm{H}$, et al: Calcium-activated chloride channel ANO1 promotes breast cancer progression by activating EGFR and CAMK signaling. Proc Natl Acad Sci USA 110: E1026-E1034, 2013. 